\title{
Long-Term, Open-Label, Safety Study of Edivoxetine as Adjunctive Treatment for Adult Patients with Major Depressive Disorder Who Were Partial Responders to Selective Serotonin Reuptake Inhibitor Treatment in Japan
}

\author{
Emel Serap Monkul', Mark Bangs ${ }^{1}$, Keita Asato², Masashi Takahashi' ${ }^{2}$, Yasushi Takita ${ }^{2}$, \\ Mary Anne Dellva', Jonna Ahl'1, Celine Goldberger'1 \\ ${ }^{1}$ Eli Lilly and Company, Indianapolis, IN, USA \\ ${ }^{2}$ Eli Lilly Japan K.K. Office, Kobe, Japan \\ Email: nery emel serap monkul@lilly.com
}

Received 3 March 2015; accepted 9 April 2015; published 13 April 2015

Copyright (C) 2015 by authors and Scientific Research Publishing Inc.

This work is licensed under the Creative Commons Attribution International License (CC BY).

http://creativecommons.org/licenses/by/4.0/

(c) (i)

\section{Abstract}

Edivoxetine is a highly selective norepinephrine reuptake inhibitor (NRI) that has been investigated in short-term studies as adjunctive therapy to selective serotonin reuptake inhibitor antidepressants (SSRIs) in patients with major depressive disorder (MDD) who were partial responders to their SSRIs. This 52-week open-label study investigated the safety and tolerability of longer-term treatment with adjunctive edivoxetine in patients with MDD in Japan, who had completed one of two placebo-controlled acute studies of edivoxetine as adjunctive therapy to SSRIs. All patients continued on their stable dose of SSRI. Two hundred eighty-eight patients were enrolled and assessed for up to 1 year using standard safety and tolerability measures. Of these, 195 patients previously received only placebo in the parent study and, therefore, were first exposed to edivoxetine in this study. Approximately $46 \%$ of patients completed the study. The most frequently cited $(>5 \%)$ reasons for discontinuation were sponsor decision $(19.4 \%$, which included patients discontinued due to early study termination), adverse event (17.4\%) and subject decision (8.7\%). Adverse events leading to discontinuation in more than 2 patients $(>1 \%)$ were palpitations, vomiting, hepatic function abnormal, hypertension, nausea, and tachycardia. Treatment-emergent elevations in diastolic blood pressure and pulse were at least twice that reported in the literature for non-Asian patients. Twenty percent of patients had sustained elevations in pulse. Treatment- 
emergent changes in laboratory measures were small and not clinically meaningful. Assessment across all safety measures in this study indicated that the safety profile of edivoxetine was consistent with that expected for a selective NRI.

\title{
Keywords
}

\author{
Edivoxetine, Depression, SSRI, NRI, Japan, Adjunctive Therapy
}

\section{Introduction}

Major depressive disorder (MDD) is a chronic illness that is estimated to have $2.9 \%$ lifetime prevalence in Japan [1]. Although the prevalence rates for MDD are lower in Japan than those reported in western countries, suicide rates in Japan are among the highest in the world with 24.4 per 100,000 inhabitants [2]. In a psychological autopsy case-control study of suicide in Japan, MDD was the disorder that was the most strongly associated with suicide, as well as anxiety disorder, alcohol-related disorders, and brief psychotic disorder [1]. As in other countries, the societal cost of depression in Japan is enormous, and was estimated to be ¥2.0 trillion in 2005 [3].

Complete remission of symptoms is the treatment goal in MDD, but is unattainable for many patients, which puts them at risk for relapse [4] and suicide [5]. First-line therapy for MDD is the most frequent monotherapy with a selective serotonin reuptake inhibitor (SSRI), though individual differences in response to treatment can contribute to incomplete symptom resolution. One second-step treatment option would be to switch to or add on another antidepressant with a different mode of action [6], and the addition of an agent with selective norepinephrine reuptake inhibition (NRI) to an SSRI antidepressant may provide additional benefit [7].

Edivoxetine is a highly selective NRI that has been investigated as adjunctive therapy to SSRI antidepressants in several acute placebo-controlled trials [8]-[10] and one open-label long-term safety trial [11] in patients with MDD who were partial responders to SSRI treatment. However, these studies were conducted in predominantly white populations. Since racial/genetic variations can contribute to differences in metabolism of psychiatric medications, which can ultimately impact efficacy and safety outcomes [12], it is important to conduct analyses in specific racial/genetic populations [13]. Herein we report on the tolerability and safety of edivoxetine as adjunctive treatment to SSRI antidepressants in a year-long, open-label trial conducted in Japan in patients with MDD who were partial responders to SSRI treatment.

\section{Methods}

This was a phase III, multicenter, open-label investigation of the long-term tolerability and safety of edivoxetine as adjunctive treatment in patients with MDD in Japan. The study was conducted at 27 sites in accordance with ethical principles that had their origin in the Declaration of Helsinki, and were consistent with Good Clinical Practices and applicable laws and regulations. The institutional review boards for each site approved the protocol and all patients provided written informed consent.

Enrollment began 2 August 2011 and the final visit occurred on 16 January 2014 (Clinical Trials.gov identifier: NCT01370499). The study planned to enroll approximately 320 patients, but was closed early because the parent studies, H9P-MC-LNBQ (NCT01187407) and H9P-MC-LNBM (NCT01173601), did not meet the primary efficacy endpoint of a statistically significant difference between adjunctive edivoxetine and adjunctive placebo on the mean change from baseline to Week 8 in the Montgomery-Asberg Depression Rating Scale (MADRS) [14] total score. At the time of study closure, 288 patients had been enrolled and assessed for up to one year from baseline.

Patients were eligible to enter the parent studies if they had been taking an SSRI that had been approved for MDD at a dose within the labeling guidelines for Japan. Duration of SSRI treatment had to be $\geq 6$ weeks before visit 2, with at least the last 4 weeks at a stable, optimized dose as determined by the investigator. Eligible patients were required to meet criteria for partial response at visits 1 and 2, as defined by the investigator's opinion that the patient had experienced at least a minimally clinically meaningful improvement with the SSRI treatment. Additionally, patients had to score $\geq 16$ on the GRID 17-Item Hamilton Depression Rating Scale total score [15] and to rate $\leq 75 \%$ improvement for their current SSRI by using the patient-rated Massachusetts General Hospital 
Antidepressant Treatment Response Questionnaire-Modified Version [16] at visit 1.

Both parent studies included three periods: screening, an 11-week double-blind treatment period [3-week placebo lead-in and 8 weeks of acute therapy for randomized patients], and a discontinuation period of 1 - 2 weeks. Throughout all study periods, patients were required to take their SSRI at a stable dose. During the double-blind placebo lead-in, those patients who had $<25 \%$ improvement on the MADRS total score and a MADRS total score $\geq 14$ at the end of the 3 weeks were randomly assigned to receive adjunctive edivoxetine or adjunctive placebo for 8 weeks. Patients who did not meet randomization criteria were kept on adjunctive placebo plus their SSRI, and were continued in the study to maintain the blind.

The studies differed with respect to dosing and discontinuation of adjunctive edivoxetine. In the LNBQ study, patients meeting randomization criteria were randomly assigned to once daily (QD) doses of adjunctive edivoxetine $6 \mathrm{mg}$ fixed-dose, adjunctive edivoxetine 12 - $18 \mathrm{mg}$ flexible dose, or adjunctive placebo. At the end of the double-blind treatment period, patients were abruptly discontinued from adjunctive edivoxetine and received adjunctive placebo for one week. In the LNBM study, patients meeting randomization criteria were randomly assigned to receive fixed doses of adjunctive edivoxetine $12 \mathrm{mg}$ QD or $18 \mathrm{mg}$ QD or placebo. At the end of double-blind treatment, study medication was discontinued over a 2-week period. Patients in both adjunctive edivoxetine groups were randomized to an abrupt or tapered discontinuation. The taper schedule was $12 \mathrm{mg}$ for 4 days, $6 \mathrm{mg}$ for 4 days, and placebo for 6 days. Patients randomly assigned to abrupt discontinuation received placebo for the entire 2 weeks. Patients who received placebo during the acute adjunctive treatment phase continued to receive placebo during the discontinuation period.

To be eligible for the current study, patients in Japan were to have completed one of the two acute phase III parent studies (including the discontinuation period and were no longer taking adjunctive medication), and were still taking the same SSRI at the optimized and stable dose as in the parent study. Since these patients had already met the inclusion and exclusion criteria at entry to the parent studies, they were not re-assessed prior to enrollment in this study. Both non-randomized and randomized patients from the parent studies were eligible.

At enrollment, all patients began treatment with oral adjunctive edivoxetine $12 \mathrm{mg}$ QD. After 1 week, the dose could be adjusted up to $18 \mathrm{mg} /$ day and then back down to $12 \mathrm{mg} /$ day if needed, based on tolerability and efficacy. At week 12, the dose could not be adjusted for the remainder of the study. At week 52 or at early discontinuation, adjunctive edivoxetine was discontinued abruptly and patients were followed up one week later. All patients were maintained on their stable dose of SSRI throughout the study including follow up after abrupt discontinuation of adjunctive edivoxetine.

The primary safety and tolerability assessments included: discontinuation rates, treatment-emergent adverse events (TEAEs), vital signs, weight, electrocardiograms (ECGs), and laboratory analysis. Secondary safety and tolerability measures included serious adverse events, adverse events that were reported as a reason for discontinuation, dose reduction, or were associated with discontinuation of adjunctive edivoxetine; suicidal ideation/behavior assessed with the Columbia-Suicide Severity Rating Scale (CSSR-S) [17], changes in sexual function assessed with the Arizona Sexual Experiences (ASEX) scale [18]; and cognitive and physical function assessed using the Massachusetts General Hospital Cognitive and Physical Functioning Questionnaire (MGHCPFQ) [19].

The C-SSRS [17] captures the occurrence, severity, and frequency of suicide-related thoughts and behaviors during the assessment period. The scale includes suggested questions to solicit the type of information needed to determine if a suicide-related thought or behavior occurred.

The ASEX [18] is a patient-rated assessment of sexual interest, arousal, response, ability to achieve orgasm, and satisfaction with orgasm that uses a 6-point scale, ranging from "extremely" (score =1) to "never" or "no" (score =6). Patients were categorized as having sexual dysfunction if they had an ASEX total score of $\geq 19$, a score of $\geq 5$ on any item; or a score of $\geq 4$ on any 3 items.

The MGH-CPFQ [19] is a patient-rated questionnaire consisting of 7 questions pertaining to a patient's cognitive and physical well-being in which each question is rated on a 6-point scale, with scores ranging from "greater than normal" (score $=1$ ) to "normal" (score $=2$ ), to "totally absent" (score $=6$ ). The MGH-CPFQ total score ranges from 7 to 42, and higher scores were associated with less cognitive and physical well-being. The scale assesses symptoms that may be either residual to the depressive illness or associated with TEAEs of antidepressant therapies for the previous month.

Triplicate measures of blood pressure and single measures of pulse were taken at each study visit in the sitting position. The three blood pressure measures were averaged for use in the analyses. Elevated blood pressure was 
defined as: systolic blood pressure (SBP) $\geq 140 \mathrm{~mm} \mathrm{Hg}$ and an increase of $\geq 20 \mathrm{~mm} \mathrm{Hg}$ from baseline; or diastolic blood pressure (DBP) $\geq 90 \mathrm{~mm} \mathrm{Hg}$ and an increase of $\geq 10 \mathrm{~mm}$ Hg from baseline. Elevated pulse was defined as $>100$ beats per minute (bpm) and an increase of $\geq 15 \mathrm{bpm}$ from baseline. Sustained elevations in blood pressure and pulse were defined by the above criteria that occurred at three consecutive visits. Potentially clinically significant changes in blood pressure were defined as: $\mathrm{SBP} \geq 180 \mathrm{~mm} \mathrm{Hg}$ and an increase $\geq 20 \mathrm{~mm} \mathrm{Hg}$ from baseline; or DBP $\geq 105 \mathrm{~mm} \mathrm{Hg}$ and an increase $\geq 15 \mathrm{~mm} \mathrm{Hg}$ from baseline.

The safety analyses were conducted on all enrolled patients who took at least one dose of adjunctive edivoxetine. For those patients who received placebo in the parent studies, this study was the first time that they received adjunctive edivoxetine, so the safety results were summarized for all patients and by the adjunctive treatment assignments in the parent studies. Patients who received adjunctive placebo in the parent studies comprised the placebo/edivoxetine (PBO/EDX) group, and those patients who received adjunctive edivoxetine in the parent studies comprised the edivoxetine/edivoxetine (EDX/EDX) group. Safety outcomes were evaluated based on adjunctive treatment assignment in the parent study. There were baseline differences between patients with prior exposure to edivoxetine (EDX/EDX) and those who had no prior exposure (PBO/EDX); however, no statistical comparisons were made between these two groups. Categorical variables were summarized with counts and percentages. Analyses of covariance (ANCOVA) models were used to analyze continuous variables, and the model contained the main effect of pooled investigative site and the baseline value as a covariate. Type III sumof-squares were used to calculate the least-squares (LS) means from the ANCOVA model. For continuous analyses, a separate model was fit for each group defined by the adjunctive treatment phase assignments from the parent studies.

\section{Results}

Patient characteristics, illness severity, and SSRI treatment are summarized in Table 1. Twice as many patients comprised the PBO/EDX group because it included patients from the parent study lead-in period who remained on placebo, in addition to those patients who were randomized to adjunctive placebo. Approximately $46 \%$ of the patients completed the study. The most frequently cited ( $>5 \%)$ reasons for discontinuation were sponsor decision (19.4\%, which included patients discontinued due to early study termination), adverse event (17.4\%) and subject decision $(8.7 \%)$.

Over the course of the study, $88.9 \%$ of all patients reported at least one TEAE and there were no deaths. The most common ( $>5 \%$ frequency) TEAEs are summarized in Table 2 . Most of the TEAEs were mild or moderate in severity.

SAEs were reported by 2 patients in the EDX/EDX group (contusion and hyperglycemia) and 1 patient in the $\mathrm{PBO} / \mathrm{EDX}$ group reported an SAE of subdural hygroma. None of these events were considered by the investigator to be associated with the investigational product or study procedure.

Table 1. Patient demographics, baseline illness severity, and selective serotonin reuptake inhibitor treatment.

\begin{tabular}{|c|c|c|c|}
\hline Variable & PBO/EDX N = 195 & EDX/EDX N = 93 & All Patients N = 288 \\
\hline Age (years), mean (SD), range & 39.3, (9.4), 21.2 - 77.8 & 37.4, (9.2), 20.6 - 64.9 & 38.7, (9.4), 20.6 - 77.8 \\
\hline Males, n (\%) & 107 (54.9) & $48(51.6)$ & $155(53.8)$ \\
\hline Females, n (\%) & $88(45.1)$ & $45(48.4)$ & $133(46.2)$ \\
\hline Japanese, n (\%) & $195(100)$ & $93(100)$ & $288(100)$ \\
\hline Weight (kg), mean (SD) & 66.3, (15.5) & $64.5,(14.4)$ & 65.7, (15.1) \\
\hline \multicolumn{4}{|l|}{ SSRI, n (\%) } \\
\hline Sertraline & $80(41.0)$ & $36(38.7)$ & $116(40.3)$ \\
\hline Paroxetine & $57(29.2)$ & $21(22.6)$ & $78(27.1)$ \\
\hline Fluvoxamine & $35(18.0)$ & $20(21.5)$ & $55(19.1)$ \\
\hline Escitalopram & $23(11.8)$ & $16(17.2)$ & 39 (13.5) \\
\hline
\end{tabular}

Abbreviations: $\mathrm{PBO}=$ placebo; EDX = edivoxetine; SD = standard deviation; SSRI = selective serotonin reuptake inhibitor. 
Table 2. Treatment-emergent adverse events that were reported by at least $5 \%$ of all patients.

\begin{tabular}{cccc}
\hline Variable, $\mathrm{n}(\%)$ & Placebo/Edivoxetine $\mathrm{N}=195$ & Edivoxetine/Edivoxetine $\mathrm{N}=93$ & All Patients $\mathrm{N}=288$ \\
\hline Patients with at least one TEAE & $176(90.3)$ & $79(85.9)$ & $255(88.9)$ \\
Nasopharyngitis & $56(28.7)$ & $29(31.5)$ & $85(29.6)$ \\
Tachycardia & $62(31.8)$ & $17(18.5)$ & $79(27.5)$ \\
Hyperhidrosis & $33(16.9)$ & $16(17.4)$ & $49(17.1)$ \\
Palpitations & $36(18.5)$ & $12(13.0)$ & $48(16.7)$ \\
Nausea & $26(13.3)$ & $8(8.7)$ & $34(11.9)$ \\
Heart rate increased & $25(12.8)$ & $7(7.6)$ & $32(11.2)$ \\
Constipation & $19(9.7)$ & $7(7.6)$ & $26(9.1)$ \\
Dizziness & $21(10.8)$ & $4(4.4)$ & $25(8.7)$ \\
Headache & $17(8.7)$ & $7(7.6)$ & $24(8.4)$ \\
Vomiting & $14(7.2)$ & $3(3.3)$ & $17(5.9)$ \\
\hline
\end{tabular}

Abbreviation: TEAE $=$ treatment-emergent adverse event.

Adverse events leading to study discontinuation in $\geq 2 \%$ patients in the PBO/EDX group were palpitations (n $=6,3.1 \%)$, vomiting $(n=5,2.6 \%)$, and hypertension $(n=4,2.1 \%)$; in the EDX/EDX group only palpitations was reported by at least $2 \%$ of the patients.

Longitudinal changes in blood pressure and pulse are shown in Figure 1. Across the study, changes in blood pressure and pulse were numerically greater in the PBO/EDX group. At the end of 52 weeks of adjunctive treatment, LS mean (SE) changes from baseline in blood pressure and pulse measures in the PBO/EDX and EDX/EDX groups, respectively, were: 6.7 (0.9) $\mathrm{mm} \mathrm{Hg}$ and 3.7 (1.3) $\mathrm{mm} \mathrm{Hg}$ for SBP; 7.6 (5.9) $\mathrm{mm} \mathrm{Hg}$ and 6.3 (1.0) $\mathrm{mm} \mathrm{Hg}$ for DBP; and 17.0 (1.2) bpm and 11.0 (1.5) bpm for pulse. A week after abrupt discontinuation of adjunctive edivoxetine LS mean (SE) changes in blood pressure and pulse measures in the PBO/EDX and EDX/EDX groups (respectively) were $0.9(0.7) \mathrm{mm} \mathrm{Hg}$ and $0.3(1.0) \mathrm{mm} \mathrm{Hg}$ for SBP; -2.2 (0.6) $\mathrm{mm} \mathrm{Hg}$ and -2.7 (0.9) $\mathrm{mm} \mathrm{Hg}$ for DBP; and -8.1 (0.7) bpm and -8.3 (1.0) bpm for pulse.

Categorical treatment-emergent changes in blood pressure and pulse are summarized in Table 3 . These changes were more frequently noticed in DBP and pulse. The percentage of patients who met criteria for an elevation in these measures at any time was higher ( $27 \%$ and $50 \%$, respectively), than that observed for sustained elevations (11\% and $20 \%$ ). Potentially clinically significant elevations were only noted for DBP in $3 \%$ of the patients.

The percentage of patients with treatment-emergent changes in weight ( $\geq 7 \%$ loss at any time) was $15 \%$ (17\% and $10 \%$ in the PBO/EDX and EDX/EDX groups, respectively). The overall frequency of treatment-emergent weight gain $\geq 7 \%$ was $6 \%$ ( $5 \%$ and $9 \%$, respectively).

Treatment-emergent changes in ECG parameters included rates of PR $<120 \mathrm{~ms}$ in $2.1 \%$ of patients overall (1.6\% of PBO/EDX and 3.3\% of EDX/EDX patients); the overall rate was $1.1 \%$ for QTC Fridericia changes that were $>450 \mathrm{~ms}$ in males and $>470 \mathrm{~ms}$ in females (1.0\% of PBO/EDX and $1.1 \%$ of EDX/EDX patients), and none of the patients had QTc $>500 \mathrm{~ms}$. Changes in heart rate $>100 \mathrm{bpm}$ and an increase $\geq 15 \mathrm{bpm}$ from baseline were observed in $29.0 \%$ of patients overall (32.5\% of PBO/EDX and 21.7\% of EDX/EDX patients). No clinically significant changes from baseline were observed in any of the other ECG parameters in either adjunctive treatment group.

Mean changes from baseline to each visit during the open-label adjunctive treatment phase were statistically significant for various chemistry, hematology, and urinalysis analytes. However, the mean changes were small and not considered clinically meaningful. During the study, $15 \%$ of the patients overall reported an adverse event as a reason for a dose reduction, and the most common events ( $\geq 5$ patients) were tachycardia $(n=11$, $3.8 \%)$ and palpitations $(\mathrm{n}=5,1.7 \%)$, both of which occurred more frequently in the PBO/EDX group $(\mathrm{n}=9$, $4.6 \%$; and $n=4,2.1 \%)$. 

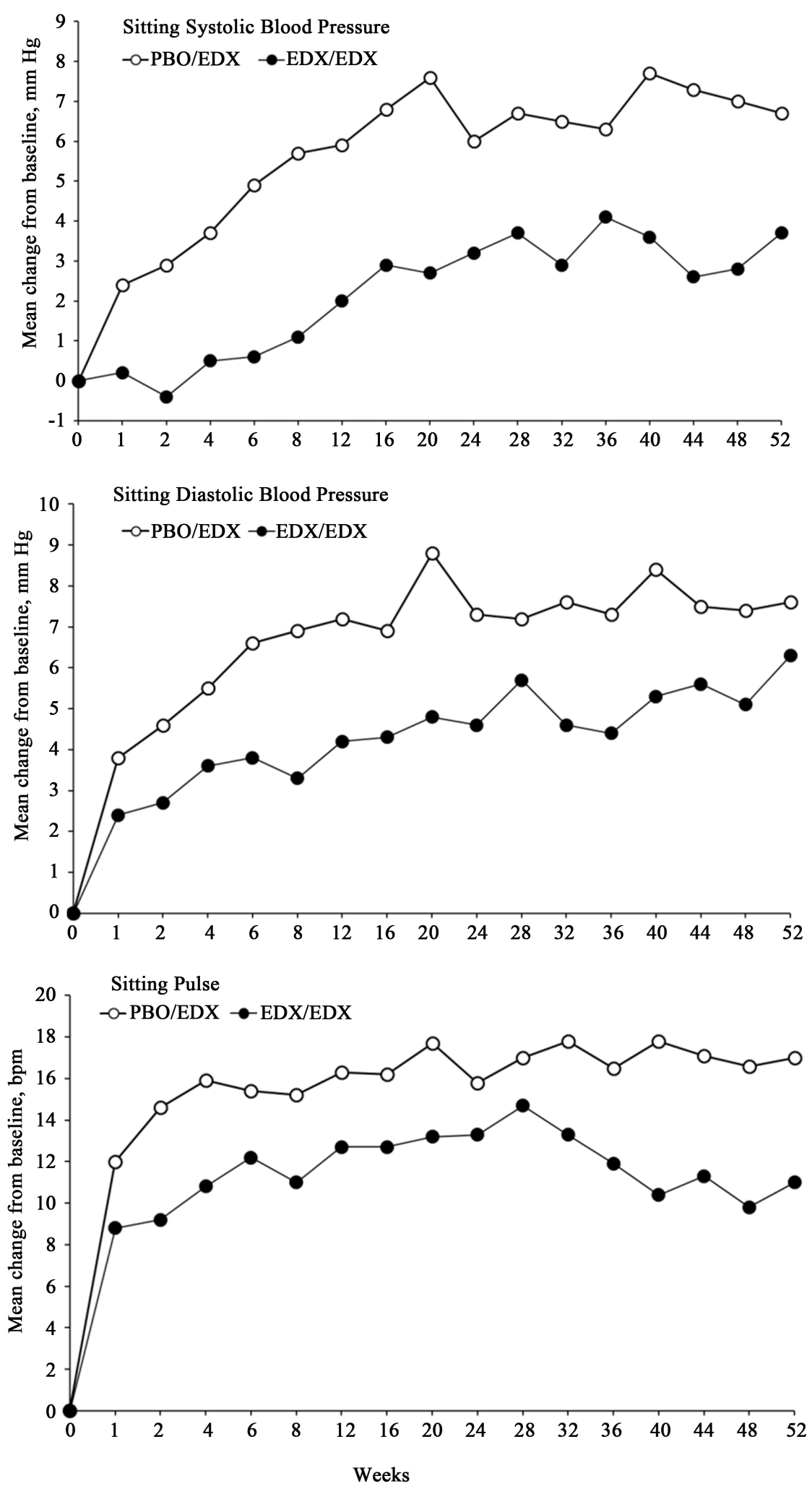

Figure 1. Least-squares mean changes from baseline in systolic blood pressure (top), diastolic blood pressure (middle) and pulse (bottom) taken while sitting at each study visit. Abbreviations: $\mathrm{PBO}=$ placebo; $\mathrm{EDX}=$ edivoxetine; $\mathrm{mm} \mathrm{Hg}=$ millimeters of mercury; bpm $=$ beats per minutes. 
Table 3. Treatment-emergent elevations in sitting blood pressure and pulse.

\begin{tabular}{|c|c|c|c|c|c|c|}
\hline \multirow[b]{2}{*}{ Measure (Unit)/Criteria } & \multicolumn{2}{|c|}{ Placebo/Edivoxetine } & \multicolumn{2}{|c|}{ Edivoxetine/Edivoxetine } & \multicolumn{2}{|c|}{ All Patients } \\
\hline & $\mathrm{N}$ & n (\%) & $\mathrm{N}$ & n (\%) & $\mathrm{N}$ & n (\%) \\
\hline \multicolumn{7}{|l|}{ Elevated Any Time } \\
\hline SDP of $\geq 140 \mathrm{~mm} \mathrm{Hg}$ and an increase of $\geq 20 \mathrm{~mm} \mathrm{Hg}$ from baseline & 195 & $20(10.3)$ & 92 & $4(4.4)$ & 287 & $24(8.4)$ \\
\hline DBP of $\geq 90 \mathrm{~mm} \mathrm{Hg}$ and an increase of $\geq 10 \mathrm{~mm} \mathrm{Hg}$ from baseline & 195 & $54(27.7)$ & 92 & $24(26.1)$ & 287 & $78(27.2)$ \\
\hline $\begin{array}{c}\text { Pulse }>100 \text { beats per minute (bpm) } \\
\text { and an increase of } \geq 15 \mathrm{bpm} \text { from baseline. }\end{array}$ & 195 & 109 (55.9) & 92 & $35(38.0)$ & 287 & $144(50.2)$ \\
\hline \multicolumn{7}{|l|}{ Sustained Elevation at 3 Consecutive Visits } \\
\hline SDP of $\geq 140 \mathrm{~mm} \mathrm{Hg}$ and an increase of $\geq 20 \mathrm{~mm} \mathrm{Hg}$ from baseline & 185 & $2(1.1)$ & 86 & 0 & 271 & $1(0.7)$ \\
\hline DBP of $\geq 90 \mathrm{~mm} \mathrm{Hg}$ and an increase of $\geq 10 \mathrm{~mm} \mathrm{Hg}$ from baseline & 185 & $22(11.9)$ & 86 & $8(9.3)$ & 271 & $30(11.1)$ \\
\hline $\begin{array}{l}\text { Pulse }>100 \text { beats per minute (bpm) } \\
\text { and an increase of } \geq 15 \mathrm{bpm} \text { from baseline }\end{array}$ & 185 & $46(24.9)$ & 86 & $8(9.3)$ & 271 & $54(20.0)$ \\
\hline \multicolumn{7}{|l|}{ Potentially Clinically Significant, Any Time } \\
\hline SDP of $\geq 180 \mathrm{~mm} \mathrm{Hg}$ and an increase of $\geq 20 \mathrm{~mm} \mathrm{Hg}$ from baseline & 195 & 0 & 92 & 0 & 287 & 0 \\
\hline DBP of $\geq 105 \mathrm{~mm} \mathrm{Hg}$ and an increase of $\geq 15 \mathrm{~mm} \mathrm{Hg}$ from baseline & 195 & $6(3.1)$ & 92 & $2(2.2)$ & 287 & $2(2.8)$ \\
\hline
\end{tabular}

Abbreviations: SDP = systolic blood pressures; DBP = diastolic blood pressure; $\mathrm{mm} \mathrm{Hg}$ = millimeters of mercury; bpm = beats per minute.

Treatment-emergent suicidal ideation or behavior as assessed by the C-SSRS was reported by $9.8 \%$ of patients overall and there was one interrupted suicide attempt in the PBO/EDX group.

The majority of patients met criteria for sexual dysfunction at baseline based on the ASEX scores: 79.6\% (121/152) of male patients and $90.2 \%$ of female patients (119/132). For patients who met criteria for sexual dysfunction at baseline, most continued to meet criteria at endpoint ( $88.4 \%$ of males and $90.8 \%$ of females). For patients who did not meet sexual dysfunction at baseline, $35.5 \%$ of male patients and $61.5 \%$ of female patients worsened and met dysfunction criteria at endpoint.

At baseline, the mean (SD) MGH-CPFQ total score was 23.8 (6.6) in the PBO/EDX group and was 25.2 (6.0) in the EDX/EDX group. At the end of the study, cognitive and physical well-being was improved with LS mean changes $(\mathrm{SE})$ from baseline that were statistically significant $(\mathrm{p} \leq 0.001)$ from zero in both groups: $-4.02(0.5)$ and -2.53 (0.8), respectively.

\section{Discussion}

This is the first long-term safety study of edivoxetine as adjunctive therapy to SSRI antidepressants inpatients with MDD in Japan, who were partial responders to their SSRI treatment. Long-term treatment with adjunctive edivoxetine in Japanese patients with MDD did not reveal safety and tolerability concerns that differed substantially from the outcomes of short-term (1 phase II and 3 phase III) adjunctive edivoxetine trials [8] [10] and a1year open-label safety and tolerability study [11]. In addition, there were no deaths during this study.

Discontinuation due to adverse events in this study (17.4\%) was higher than in the short-term adjunctive edivoxetine studies $(4.9 \%$ - 5.4\%) [8] [10], but was similar to the rate reported in the other long-term study of adjunctive edivoxetine (17.0\%) [11]. The most common adverse events ( $\geq 2 \%$ frequency) leading to early discontinuation were palpitations, vomiting and hypertension in this study; the other long-term study reported hypertension (2.0\%) [11]. The most common ( $\geq 5 \%)$ TEAEs reported in this study were similar to those reported in the previous studies: nasopharyngitis, tachycardia, hyperhidrosis, nausea, palpitations, and increased heart rate [8] [10]. Adverse events ( $\geq 5$ patients) that led to a dose reduction were palpitations and tachycardia in this study; and erectile dysfunction, nausea, and hyperhidrosis in the other long-term study [11].

Mean increases in blood pressure and pulse were observed across visits during this study. These increases were observed in the previous adjunctive edivoxetine studies [8] [10], and were expected given the mechanism of action of edivoxetine as a norepinephrine reuptake inhibitor. However, the mean increases in pulse rate and DBP was higher in this study as compared with the previous short- and long-term studies of adjunctive edivox- 
etine [8] [10] [11]. In a pooled analysis of the three phase III trials [10], there was a significant treatment-bygeographic region interaction (across Japan, the UK and the US) with adjunctive edivoxetine that was observed with changes in DBP and pulse. The interaction suggested that the hemodynamic response to NRIs as adjunctive treatment to SSRIs in Japanese patients may be different from that in non-Japanese. However, in the 1-year open-label safety study, ethnic differences in vital sign changes were not observed probably because the study population was mostly comprised of Caucasian patients [11].

It is unclear why treatment with edivoxetine as adjunctive treatment to SSRI antidepressants would be associated with higher increases in DBP and pulse in Japanese patients than that observed in Caucasian patients. In a short-term safety study of edivoxetine given as single or multiple doses that was conducted in healthy adult male Japanese and Caucasian patients (unpublished), greater increases in pulse and blood pressure were observed in the Japanese patients relative to the Caucasian patients. However, the trends of the changes were similar between the groups, and there were no significant differences between the Japanese and Caucasian patients in pharmacokinetics (PK) or pharmacodynamic (PD) evaluations (unpublished). However, PK and PD evaluations were not done in this study, so it was not known if the outcome of these measures would be different when edivoxetine was given with SSRI antidepressants.

There are few long-term studies of treatment with NRI drugs in Japan. However, there is one long-term (58 weeks) safety study of treatment with the NRI atomoxetine that is conducted in adult Japanese patients with attention deficit hyperactivity disorder (ADHD) [20]. Similarly, this study reported treatment-emergent increases in pulse (9 bpm), and systolic and diastolic blood pressure (4 $\mathrm{mm} \mathrm{Hg}$ and $5 \mathrm{~mm} \mathrm{Hg}$, respectively). Because these vital sign changes were similar to those reported in long-term studies in North American adults with ADHD [21]-[23], they were considered to be consistent with the known NRI mode of action.

The open-label study design and lack of control group limit the interpretation of observed changes that may have been associated with non-treatment effects. In addition, there were no statistical comparisons performed between groups and only categorical variables were summarized with counts and percentages. However, the open-label design mirrored clinical practice and provided a description of key clinically relevant safety outcomes in Japanese patients.

In conclusion, the assessment across all safety measures in the long-term treatment with edivoxetine as adjunctive therapy to SSRI antidepressants in Japanese patients with MDD indicated that the safety profile of edivoxetine was consistent with that expected for a selective NRI.

\section{Acknowledgements}

This work was supported by Eli Lilly and Company, Indianapolis, IN, USA.

\section{References}

[1] Kawakami, N., Takeshima, T., Ono, Y., Uda, H., Hata, Y., Nakane, Y., et al. (2005) Twelve-Month Prevalence, Severity, and Treatment of Common Mental Disorders in Communities in Japan: Preliminary Finding from the World Mental Health Japan Survey 2002-2003. Psychiatry and Clinical Neuroscience, 59, 441-452. http://dx.doi.org/10.1111/j.1440-1819.2005.01397.x

[2] Värnik, P. (2012) Suicide in the World. International Journal of Environmental Research and Public Health, 9, $760-771$. http://dx.doi.org/10.3390/ijerph9030760

[3] Sado, M., Yamauchi, K., Kawakami, N., Ono, Y., Furukawa, T.A., Tsuchiya, M., et al. (2011) Cost of Depression among Adults in Japan in 2005. Psychiatry and Clinical Neuroscience, 65, 442-450. http://dx.doi.org/10.1111/j.1440-1819.2011.02237.x

[4] Bakish, D. (2001) New Standard of Depression Treatment: Remission and Full Recovery. The Journal of Clinical Psychiatry, 62, S5-S9.

[5] McGirr, A., Séguin, M., Renaud, J., Benkelfat, C., Alda, M. and Turecki, C. (2006) Gender and Risk Factors for Suicide: Evidence for Heterogeneity in Predisposing Mechanisms in a Psychological Autopsy Study. Journal of Clinical Psychiatry, 67, 1612-1617. http://dx.doi.org/10.4088/JCP.v67n1018

[6] Trivedi, M.H., Rush, A.J., Wisniewski, S.R., Nierenberg, A.A., Warden, D., Ritz, L., et al. (2006) Evaluation of Outcomes with Citalopram for Depression Using Measurement-Based Care in STAR*D: Implications for Clinical Practice. The American Journal of Psychiatry, 163, 28-40. http://dx.doi.org/10.1176/appi.ajp.163.1.28

[7] Lucca, A., Serretti, A. and Smeraldi, E. (2000) Effect of Reboxetine Augmentation in SSRI Resistant Patients. Human Psychopharmacology: Clinical and Experimental, 15, 143-145. 
http://dx.doi.org/10.1002/(SICI)1099-1077(200003)15:2<143::AID-HUP152>3.0.CO;2-N

[8] Ball, S., Dellva, M.A., D’Souza, D.N., Marangell, L.B., Russell, J.M. and Goldberger, C. (2014) A Double-Blind, Placebo-Controlled Study of Edivoxetine as an Adjunctive Treatment for Patients with Major Depressive Disorder Who Are Partial Responders to Selective Serotonin Reuptake Inhibitor Treatment. Journal of Affective Disorders, 167, 215-223. http://dx.doi.org/10.1016/j.jad.2014.06.006

[9] Ball, S.G., Ferguson, M.B., Martinez, J.M., Pangallo, B.A., Nery, E.S., Dellva, M.A., Sparks, J., Liu, P., Zhang, Q. and Bangs, M. (in Press) Efficacy Outcomes from 3 Clinical Trials of Edivoxetine as Adjunctive Treatment for Patients with Major Depressive Disorder who are Partial Responders to Selective Serotonin Reuptake Inhibitor Treatment. Unpublished. Journal of Clinical Psychiatry.

[10] Martinez, J.M., Ferguson, M.B., Pangallo, B.A., Oakes, T.M., Sparks, J., Dellva, M.A., Zhang, Q., Liu, P., Bangs, M.E., Ahl, J. and Goldberger, C. (in Press) Safety and Tolerability of Edivoxetine as Adjunctive Treatment to Selective Serotonin Reuptake Inhibitor Antidepressants for Patients with Major Depressive Disorder. Drugs in Context.

[11] Ball, S., Atkinson, S., Sparks, J., Bangs, M., Goldberger, C. and Dubé, S. (2015) Long-term, Open-label, Safety Study of Edivoxetine 12 to $18 \mathrm{mg}$ Once Daily as Adjunctive Treatment for Patients with Major Depressive Disorder Who Are Partial Responders to Selective Serotonin Reuptake Inhibitor Treatment. Journal of Clinical Psychopharmacology (in Press).

[12] Malhotra, A.K., Myrphym Jr., G.M. and Kennedy, J.L. (2004) Pharmacogenetics of Psychotropic Drug Response. American Journal ofPsychiatry, 161, 780-796. http://dx.doi.org/10.1176/appi.ajp.161.5.780

[13] Geller, S.E., Koch, A., Pellettier, B. and Carnes, M. (2011) Inclusion, Analysis, and Reporting of Sex and Race/Ethnicity in Clinical Trials: Have We Made Progress? Journal of Women’s Health, 20, 315-320. http://dx.doi.org/10.1089/jwh.2010.2469

[14] Montgomery, S.A. and Åsberg, M. (1979) A New Depression Scale Designed to be Sensitive to Change. The British Journal of Psychiatry, 134, 382-389. http://dx.doi.org/10.1192/bjp.134.4.382

[15] Williams, J.B., Kobak, K.A., Bech, P., Engelhardt, N., Evans, K., Lipsitz, J., et al. (2008) The GRID-HAMD: Standardization of the Hamilton Depression Rating Scale. International Clinical Psychopharmacology, 23, 120-129. http://dx.doi.org/10.1097/YIC.0b013e3282f948f5

[16] Chandler, G.M., Iosifescu, D.V., Pollack, M.H., Targum, S.D. and Fava, M. (2010) Research: Validation of the Massachusetts General Hospital Antidepressant Treatment History Questionnaire (ATRQ). CNS Neuroscience and Therapeutics, 16, 322-325. http://dx.doi.org/10.1111/j.1755-5949.2009.00102.x

[17] Posner, K., Brown, G.K., Stanley, B., Brent, D.A., Yershova, K.V., Oquendo, M.A., et al. (2011) The Columbia-Suicide Severity Rating Scale: Initial Validity and Internal Consistency Findings from Three Multisite Studies with Adolescents and Adults. The American Journal of Psychiatry, 168, 1266-1277. http://dx.doi.org/10.1176/appi.ajp.2011.10111704

[18] McGahuey, C.A., Gelenberg, A.J., Laukes, C.A., Moreno, F.A., Delgado, P.L., McKnight, K.M., et al. (2000) The Arizona Sexual Experience Scale (ASEX): Reliability and Validity. Journal of Sex and Marital Therapy, 26, 25-40. http://dx.doi.org/10.1080/009262300278623

[19] Fava, M., Iosifescu, D.V., Pedrelli, P. and Baer, L. (2009) Reliability and Validity of the Massachusetts General Hospital Cognitive and Physical Functioning Questionnaire. Psychotherapy and Psychosomatics, 78, 91-97. http://dx.doi.org/10.1159/000201934

[20] Hirata, Y., Goto, T., Takita, Y., Trzepacz, P.T., Allen, A.J., Ichikawa, H. and Takahashi, M. (2014) Long-Term Safety and Tolerability of Atomoxetine in Japanese Adults with Attention Deficit Hyperactivity Disorder. Asia-Pacific Psychiatry, 6, 292-301. http://dx.doi.org/10.1111/appy.12119

[21] Adler, L.A., Spencer, T.J., Williams, D.W., Moore, R.J. and Michelson, D. (2008) Long-Term, Open-Label Safety and Efficacy of Atomoxetine in Adults with ADHD: Final Report of a 4-Year Study. Journal of Attention Disorders, 12, 248-253. http://dx.doi.org/10.1177/1087054708316250

[22] Adler, L.A., Spencer, T., Brown, T.E., Holdnack, J., Saylor, K., Schuh, K., et al. (2009) Once-Daily Atomoxetine for Adult Attention-Deficit/Hyperactivity Disorder: A 6-Month, Double-Blind Trial. Journal of Clinical Psychopharmacology, 29, 44-50.

[23] Young, J.L., Sarkis, E., Qiao, M. and Wietecha, L. (2011) Once-Daily Treatment with Atomoxetine in Adults with Attention-Deficit/Hyperactivity Disorder in Adults: A 24-Week, Randomized, Double-Blind, Placebo-Controlled Trial. Clinical Neuropharmacology, 34, 51-60. 